\title{
BMJ Open Apical extrusion of debris with different rotary and reciprocating single-file endodontic instrumentation systems: a systematic review and meta- analysis protocol
}

To cite: Ahmad MZ, Sadaf D, MacBain MMcC, et al. Apical extrusion of debris with different rotary and reciprocating single-file endodontic instrumentation systems: a systematic review and metaanalysis protocol. BMJ Open 2020;10:e038502. doi:10.1136/ bmjopen-2020-038502

- Prepublication history for this paper is available online. To view these files, please visit the journal online (http://dx.doi org/10.1136/bmjopen-2020038502).

Received 12 March 2020

Revised 16 April 2020

Accepted 09 July 2020

\section{Check for updates}

(c) Author(s) (or their employer(s)) 2020. Re-use permitted under CC BY-NC. No commercial re-use. See rights and permissions. Published by BMJ.

${ }^{1}$ Department of Restorative Dentistry, College of Dentistry in Ar Rass, Qassim University, Ar Rass, Saudi Arabia

${ }^{2}$ Nuffield Department of Primary Care Health Sciences, Centre for Evidence-Based Medicine, University of Oxford, Oxford, UK ${ }^{3}$ Department of Conservative Sciences, College of Dentistry, Qassim University, Buraydha, Saudi Arabia

Correspondence to Muhammad Zubair Ahmad; m.muhammad@qu.edu.sa

\section{ABSTRACT}

Introduction Root canal treatment is one of the oldest dental procedures for the treatment of endodontic infection. Extrusion of debris beyond the root apex during root canal instrumentation and subsequent persistence of pain are common complications. A systematic review of the evidence on reciprocating single-file instrumentation systems and their comparison with rotary single-file systems, with apical extrusion of debris as primary outcome, will be done through this study.

Methods and analysis Published ex vivo and in vitro studies with no language restriction will be included. We will search MEDLINE (Ovid), EMBASE (Ovid), Web of Science, Cochrane and Google Scholar. Strategies will be incorporated to search grey literature also. Thorough evaluation of search results, completion of data abstraction and assessment of quality will be done by two reviewers independent from each other. Assessment of included studies will be done by utilising an evidence model developed on the basis of standards of quality reported in guidelines to document ex vivo and in vitro studies regarding dental materials and pertained for extrusion of debris apically and has been already used in quality assessment of studies involving quantification of debris extrusion apically. We will calculate the standardised mean differences for apically extruded debris, with congruent 95\% Cls. We will measure the statistical heterogeneity by applying the Cochrane $Q$ test and quantify using the $\mathrm{I}^{2}$ statistic. Existence of covariates and any potential heterogeneity will be explored through prespecified subgroup and sensitivity analyses.

Ethics and dissemination Approval from an ethical research committee is not required because it will be done using data that have been already published and have no concerns related to the privacy of patients. Extensive dissemination of results from this review will be done through submission to a peer-reviewed journal for publication and conferences.

PROSPERO registration number CRD42019151804.

\section{INTRODUCTION}

Root canal treatment (RCT), in modern dentistry, is one the most frequently
Strengths and limitations of this study

- This review will comprehensively examine singlefile rotary systems that work with full rotation-type movement in comparison to single-file rotary systems that work with reciprocating-type motion.

- An extensive literature search, developed in consultation with information specialist and librarian with broad experience in systematic reviews, will be conducted to include studies from multiple databases, without language and publication type restrictions.

- Prespecified subgroup analysis, planned in consultation with medical statistician expert in statistical planning and analysis of systematic reviews and meta-analysis, will be executed.

- This protocol does not plan to evaluate multiple-file endodontic instrumentation systems.

performed invasive procedures. This procedure is routinely performed by general dentists and specialist endodontists both to treat root canal infections and also to preserve the natural tooth. Despite drastically improved success rate of RCT since its inception in the 17 th century, ${ }^{12}$ patient complications remain a persistent challenge, the most common of which is postoperative pain. ${ }^{3-5}$ Postoperative pain in root canal-treated patients is classically reported as ranging anywhere from mild to severe in intensity. ${ }^{6}$ Moderate to severe postoperative pain can severely impact patient well-being and may require emergency dental visits and, potentially, prescription of analgesic medications or more invasive endodontic retreatment. ${ }^{7}$

It was postulated that postoperative pain occurs as a result of extrusion of debris beyond the root canal apex during root canal preparation. ${ }^{89}$ This apical extrusion of debris causes acute inflammatory reaction due to localised 
irritation and disruption of balance between root canal microbiota and host defence during root canal instrumentation. ${ }^{10}$ Root canal preparation comprises chemomechanical preparation. This includes canal shaping with mechanical means and cleaning procedures using chemical irrigants inside the root canal space to achieve proper disinfection. During root canal preparation debris that may also contain bacteria, necrotic pulp tissue and dentine can be introduced into the periapical tissues. ${ }^{11}$ It is possible that certain factors can be controlled by the operators such as selection of endodontic file systems and technique of root canal preparation but virulence of the microorganisms extruded beyond apex is almost impossible to control. ${ }^{12} 13$ Endodontic instruments are continuously evolving from hand instruments to enginedriven rotary and reciprocating as well as from multiplefile to single-file systems. Despite various techniques and improvements in the design of instruments for root canal preparation and irrigation, extrusion of debris and irrigants beyond the apical foramen remains a challenge. Of prime importance is the type of motion of instruments. Endodontic single-file instruments can be classified into two groups based on the type of motion: continuous rotating and reciprocating files. ${ }^{14}$

Two of these newer rotary endodontic systems, OneShape (MicroMega, Besancon, France) and Neoniti (Neolix, Châtres-la-Forêt, France), work in a continuous rotation manner and are designed to prepare the root canal with a single instrument only. The Reciproc (VDW, Munich, Germany) and WaveOne (Dentsply Maillefer, Ballaigues, Switzerland) are also designed to prepare the root canal with single instrument but with the use of reciprocating motion with individual automated devices. Mettalurgical improvements and heat treatment have resulted in endodontic files with improved characteristics and shaping performance. WaveOne Gold (Dentsply Maillefer, Ballaigues, Switzerland) and Reciproc blue (VDW, Munich, Germany) are two such important improved systems. ${ }^{15}$

It is claimed that the reciprocating single-file systems such as Reciproc, Reciproc blue, WaveOne and WaveOne Gold can prepare and clean root canals completely using only one instrument and with minimal risk of extrusion of debris beyond the root apex. It is thought that root canal preparation using instruments with reciprocatingtype motion is a progression of the balanced force technique, which permits the shaping of root canals to a larger apical diameter using hand instruments. ${ }^{16}$ Meanwhile, it is assumed that engine-driven rotary instruments with full rotation-type motion tend to produce less apically extruded debris, as such instruments have a tendency to pull the debris into their flutes, leading the debris coronally to come out of the root canal space. ${ }^{13}$ Neoniti is made of a controlled memory wire ${ }^{1718}$ whereas OneShape is made of electropolished and surface-treated conventional austenite $55 \mathrm{Ni}$-Ti alloy. ${ }^{18}{ }^{19}$ Hyflex EDM 'one file' (Coltene/ Whaledent, Alstatten, Switzerland) is a multitapered rotary file allowing enhanced resistance to cyclic fatigue because of heat treatment and a proprietry manufacturing process. ${ }^{20}$ XP Shaper (FKG, La Chaux de Fonds, Switzerland) is a minimal taper single-file rotary system which allows for three-dimensional shaping and cleaning of the root canal space. ${ }^{21}$

\section{Objectives}

This article aimed to describe the protocol to systematically examine single-file rotary systems that work with full rotation-type motion in comparison to single-file rotary systems that work with reciprocating-type motion.

\section{Focussed question}

For teeth in which endodontic instrumentation was done using single-file systems, what is the difference in the apical extrusion of debris between rotary and reciprocating systems?

\section{METHODS AND ANALYSIS}

The Preferred Reporting Items for Systematic reviews and Meta-Analysis Protocols guidelines were followed to develop the protocol of this systematic review and metaanalysis. ${ }^{22}$ This protocol is registered with the International Prospective Register of Systematic Reviews.

\section{Literature search}

We will conduct a comprehensive search of the following databases: MEDLINE (Ovid), EMBASE (Ovid), Web of Science, Cochrane and Google Scholar, without any restrictions on language, date and type of publication. Medical subject heading terms and keywords specifically related to types of movements of single-file engine-driven endodontic instruments (reciprocating or full rotation) will be used. We will consult with a librarian with systematic review expertise for building a comprehensive search strategy. ${ }^{23}$ Table 1 shows the detailed search proposed strategy for MEDLINE (Ovid) database. Manual screening of references related to articles that will be found relevant, revision of the proceedings of meetings that will be found pertinent and communications with clinical experts in the field will be done, which will supplement our search.

\section{Study selection}

Evaluation of studies for eligibility will be done by two reviewers (MZA and DS) independent from each other. Any disagreement between the two investigators regarding the final decision about inclusion or exclusion of a study will be settled by consensus, and if necessary, consultation with a third investigator (ANM).

The inclusion criteria are the following: study design: ex vivo and in vitro studies without any restrictions on language and publication type; population: permanent teeth with mature apices of adult humans undergoing root canal preparation; intervention: root canal preparation with a single-file engine-driven instrumentation system with full rotation-type motion (OneShape, Neoniti, F6 Skytaper, Hyflex EDM 'One File', XP Shaper, One Curve, Prodesign, Edge Endo X7 and/or F360); 
Table 1 Search strategy for the MEDLINE electronic database using the Ovid interface

\begin{tabular}{|c|c|}
\hline Database & Search terms \\
\hline $\begin{array}{l}\text { MEDLINE } \\
\text { 1946-present }\end{array}$ & $\begin{array}{l}\text { 1. (Apical extrusion or debris extrusion or detritus extrusion or apical debris or extruded debris).mp.(mp=title, } \\
\text { abstract, original title, name of substance word, subject heading word, floating sub-heading word, keyword } \\
\text { heading word, protocol supplementary concept word, rare disease supplementary concept word, unique } \\
\text { identifier, synonyms) } \\
\text { 2. (Single file or single file system or Waveone or Wave one gold or reciproc or reciproc blue or predesign } \\
\text { or predesign r or X1 blue or single file endodontic or reciprocating file or reciprocating or symmetric } \\
\text { movement).mp.(mp=title, abstract, original title, name of substance word, subject heading word, } \\
\text { floating sub-heading word, keyword heading word, protocol supplementary concept word, rare disease } \\
\text { supplementary concept word, unique identifier, synonyms) } \\
\text { 3. (Rotary file or rotary instrument or rotary nickel titanium or rotary niti or niti rotary instrument or endodontic } \\
\text { rotary file or Neo-niti or Neoniti or Oneshape or F6 skytaper or Hyflex EDM or EDM or Hyflex EDM One } \\
\text { File or One file or One curve or Prodesign or predesign logic or XP shaper or edge endo X7 or edge endo } \\
\text { or F360 or continuous movement).mp.(mp=title, abstract, original title, name of substance word, subject } \\
\text { heading word, floating sub-heading word, keyword heading word, protocol supplementary concept word, } \\
\text { rare disease supplementary concept word, unique identifier, synonyms) } \\
\text { 4. } 1 \text { and } 2 \\
\text { 5. } 1 \text { and } 3 \\
6 \text { or } 4\end{array}$ \\
\hline
\end{tabular}

control: root canal preparation with a single-file enginedriven instrumentation system with reciprocating-type motion (WaveOne, WaveOne Gold, Reciproc, Reciproc blue, Prodesign R and/or X1 blue); outcome: extrusion of debris apically. If there are studies published more than once, we will consider only the reports that contain comprehensive and most informative data. Relevant data will be extracted from the included studies. We will list excluded studies in a separate table with reasons of their exclusion. The procedure for study selection is shown in figure 1 .

The exclusion criteria are the following: articles studying file systems different from OneShape, Neoniti, F6 Skytaper, Hyflex EDM 'One File', XP Shaper, One Curve, Prodesign, Edge Endo X7 and/or F360 versus WaveOne, WaveOne Gold, Reciproc, Reciproc blue, Prodesign R and/or X1 blue, or not following single-file system protocol; articles studying the influence of irrigants on the apical extrusion of debris; studies using artificial teeth to report apical extrusion of debris; studies on apical extrusion of debris in retreatment.

\section{Data management and collection}

From all the relevant databases, we will export the results of the literature search containing the complete references as .ciw, .nbib, .enw or .ris files. For reference management, EndNote X9 software will be used. Piloting of the screening questions and developing of forms will be done by reviewers. This will be based on the eligibility criteria. Full-text articles of all eligible studies will be retrieved prior to abstraction of data. Full articles will be translated into English for studies published in any other language. Additionally, in data management, a medical expert with original language fluency will be involved. Titles and abstracts, which will be retrieved through the process of comprehensive search against the eligibility criteria that will be predefined, will be independently screened by the two primary reviewers. Full texts for all the references appearing to meet the eligibility criteria will be obtained, or if there will be any ambiguity. Then, full texts will be screened by the reviewers for all references, and decision will be made regarding eligibility of these articles. Authors of the relevant studies will be contacted where necessary to retrieve additional information to resolve queries related to eligibility of the studies. If there will be any discrepancy, it will be resolved via discussion and consensus, and consultation with the third reviewer will be done if needed. For studies that will be excluded, we will record the reasons for exclusion.

Two primary reviewers (MZA and DS) will independently abstract the data that will be authenticated for accuracy by the third reviewer (ANM). Information will be gathered from the eligible studies by using the forms for data abstraction, which will include fields for type of studies, author, journal details and publication year, use of natural teeth, sample size, root canal preparation systems used, methods used to quantify apically extruded debris, representativeness of sample/comparable study groups, working length, irrigant used, reproducible methodology, standardisation of diameter of apical foramen, randomisation, blinding, control of bias, statistical analysis performed with confidence level/p-value and evidence level.

\section{Risk of bias in individual studies}

Quality assessment will be performed by two reviewers independently. Risk of bias/quality assessment will be done using an evidence model developed on the basis of quality standards documented in guidelines for reporting preclinical in vitro studies related to dental materials and implemented for debris extrusion apically and has been already used in quality assessment of ex vivo and in vitro studies involving quantification of debris extrusion apically. ${ }^{122425}$ Hence, we consider this as a valid tool 


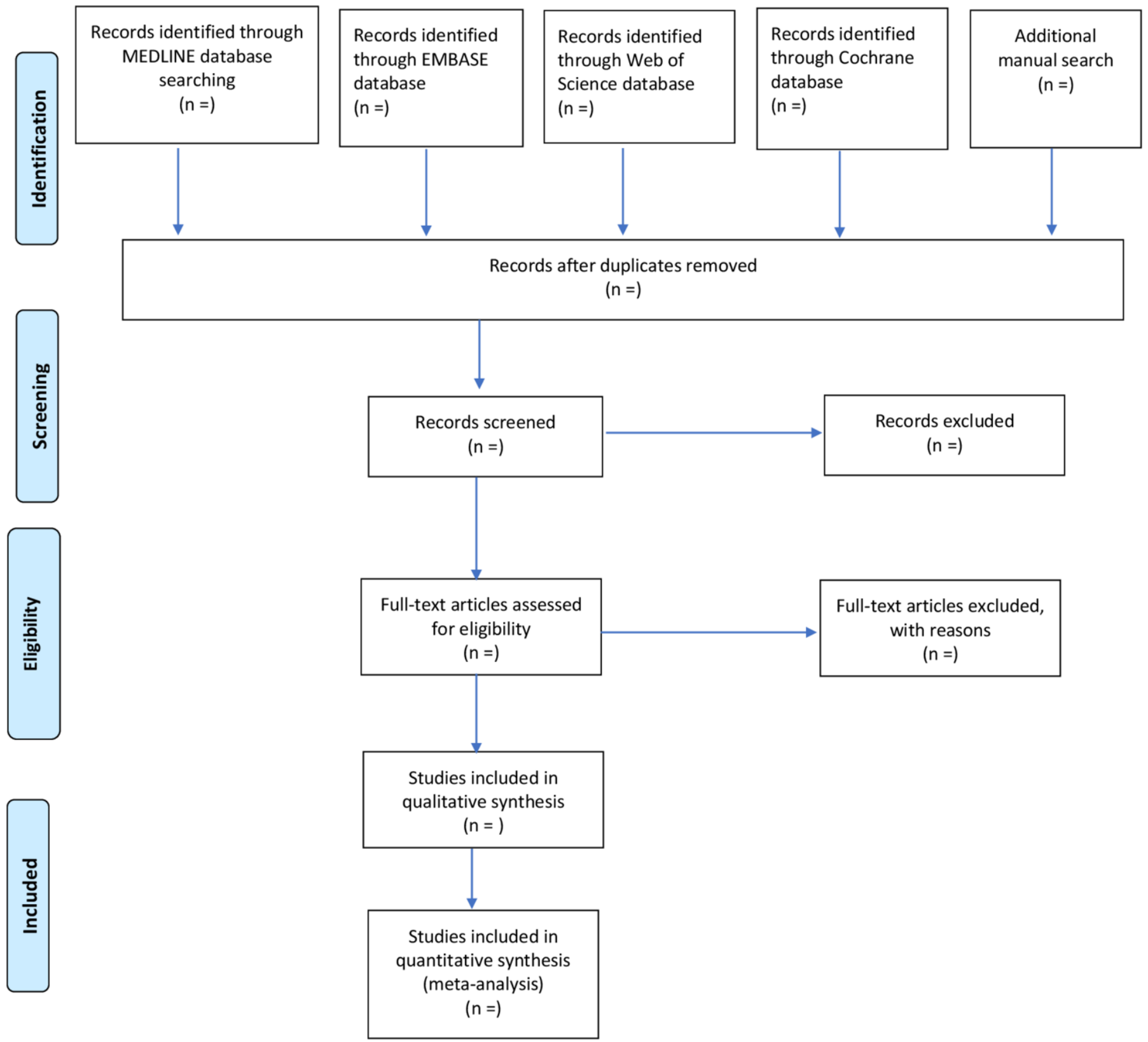

Figure 1 Flow diagram of the study selection process.

for risk of bias/quality assessment of studies that will be included in our systematic review (table 2). If sufficient information is not provided, and if any judgement is difficult, we will categorise the study as subjected to an unclear risk of bias. In such situations, we will contact the authors of the original study for more required information. We will record the data using a checklist to categorise the level of evidence. Score will be given to each domain depending on whether a specific criterion has been met or not (eg, 0 for 'unclear' or 'no' and 1 for 'yes'). A study will be considered of 'low methodological quality' if it scores 1-4 points, of 'moderate methodological quality' if it scores 5-7 points and of 'high methodological quality' if it scores $8-10$ points, in accordance with the definitions outlined in a previous study. ${ }^{24}$
Discussion, consensus and, if required, a third reviewer (ANM) will be consulted to resolve any disagreement during quality assessment.

\section{Subgroup analysis}

The two main groups of engine-driven endodontic instrumentation systems in the study will be single-file rotary and reciprocating systems, and within each group, different subgroups will be further analysed.

For the groups of rotary and reciprocating systems, all single-file instrument systems will be analysed. Once the selection of studies has been completed, each single-file instrument system in rotational type of motion group will be analysed against each single-file instrument system in reciprocating type of motion group. 
Table 2 Risk of bias assessment or assessment of quality of studies

\section{Yes/no/not}

Criteria for risk of bias assessment reported Score

1. Standardised method for quantification of apical debris

2. Natural teeth (anatomy may be extrapolated to a clinical situation)

3. Working length $-1 \mathrm{~mm}$ from apical foramen

4. Blind study

\section{Use of distilled water as irrigant}

6. Standardised diameter of the apical foramen

7. Control group

8. Randomised sample

\section{Reproducible methodology}

10. Statistical analysis carried out

\section{Total score}

\section{$8-10$, too low risk of bias.}

$5-7$, low to moderate risk of bias.

$1-4$, high risk of bias.

\section{Definition and quantification of outcome}

Our outcome will include extrusion of debris apically after root canal instrumentation expressed in units of weight (grams). It was first verified by Chapman et $a t^{26}$ that during instrumentation, infective material may be expelled from the root canal. Vande Visse and Brilliant ${ }^{27}$ confirmed later that instrumentation and irrigation of the root canal system result in collectable debris. Irrigation, however, is considered an essential component for the success of RCT, and it is highly likely that other potential risks such as persistence of infection and failure of RCT will ensue if proper irrigation was not done. ${ }^{10} 12$ Since apically extruded materials also contain irrigant, it is necessary to remove irrigant to quantify extruded debris accurately. Extruded material is collected and stored in an incubator. After evaporation of fluids, a mass of debris is obtained, which can then be measured using a measuring device, for example, an electronic microbalance in units of weight.

\section{Data synthesis}

We will compare the apical extrusion of debris for rotary single-file instruments with full rotation-type motion versus single-file instruments with reciprocating-type motion. Random-effect forms of meta-analyses will be assessed. A fixed-effects model assumes that the laboratory protocols and measurement scales are similar for all studies, whereas a random-effects meta-analysis incorporates both between-study variation and within-study variation. ${ }^{28}$ Because our outcome variable is continuous, the standardised mean difference, also known as Cohen's $d$ or effect size, will be used, and the respective $95 \%$ CI will be calculated as a measure of effect size. It will be computed with the following formula. ${ }^{29}$

$$
d=\frac{\text { (rotary system mean }- \text { reciprocating system mean })}{\text { pooled SD }} .
$$

The guidelines as suggested by Cohen will be used for interpreting the magnitude of the effect size: small $d<0.5$, moderate $d \geq 0.5$ and large $d \geq 0.8 .^{30}$ The results of the included studies will be presented in forest plots.

Tests of heterogeneity will be conducted using $Q$ statistic, which is distributed as a $\chi^{2}$ variate (assumption of homogeneity of effect sizes). The between-study heterogeneity will be assessed with the $\mathrm{I}^{2}$ statistic, with $\mathrm{I}^{2}<25 \%$, no heterogeneity; $\mathrm{I}^{2}<50 \%$, low heterogeneity, $\mathrm{I}^{2}<75 \%$, moderate heterogeneity; $\mathrm{I}^{2} \geq 75 \%$, high heterogeneity. ${ }^{32} 33$ P-values $<0.05$ will be considered statistically significant. Meta-analyses will be performed using Stata V.16.0 (StataCorp, College Station, TX, USA). Publication bias will be investigated graphically using funnel plots-plots of effect estimates against sample size. ${ }^{33} 34$

\section{Ethics and dissemination}

Approval from an ethical research committee is not required because the study will be done using data that have been already published and have no concerns related to the privacy of patients. It is expected that the results of this study will change the clinical practice significantly. By examining comprehensively the evidence on rotary single-file instruments with full rotation-type motion in comparison to single-file instruments with reciprocatingtype motion, this review will inform clinicians on which single-file rotary system to use for root canal preparation, which, in turn, will minimise complications. From this review, we will submit the results for publication to a peerreviewed journal.

\section{Twitter Muhammad Zubair Ahmad @zubairahmadd}

Acknowledgements The authors thank Mrs. Nia Roberts, the information specialist and librarian at Bodleian Libraries, University of Oxford, Oxford, UK, for her valuable support in the selection of databases and in building a comprehensive search strategy. The authors also thank Mr. Jose Ordonez-Mena, the medical statistician at the University of Oxford, Oxford, UK, for his valuable support in the planning of data synthesis.

Contributors MZA and DS conceived and designed the protocol. MZA and DS developed the search strategy and piloted it in all relevant databases. MZA, DS and MMM developed the review protocol, selection criteria, risk of bias assessment strategy and data management and synthesis methodology. MMM and ANM critically revised and commented on the intellectual content of the manuscript.

Funding The authors have not declared a specific grant for this research from any funding agency in the public, commercial or not-for-profit sectors.

\section{Competing interests None declared.}

Patient and public involvement Patients and/or the public were not involved in the design, or conduct, or reporting or dissemination plans of this research.

Patient consent for publication Not required.

Provenance and peer review Not commissioned; externally peer reviewed.

Open access This is an open access article distributed in accordance with the Creative Commons Attribution Non Commercial (CC BY-NC 4.0) license, which permits others to distribute, remix, adapt, build upon this work non-commercially, and license their derivative works on different terms, provided the original work is properly cited, appropriate credit is given, any changes made indicated, and the use is non-commercial. See: http://creativecommons.org/licenses/by-nc/4.0/. 


\section{ORCID iDs}

Muhammad Zubair Ahmad http://orcid.org/0000-0002-8033-6254

Durre Sadaf http://orcid.org/0000-0003-4504-6267

\section{REFERENCES}

1 Castellucci A. A brief history of endodontics. Italy: Endodontics Prato, 2004: 2-5.

2 Cruse WP, Bellizzi R. A historic review of endodontics, 1689-1963, part 1. J Endod 1980;6:495-9.

3 Grossman LI. A brief history of endodontics. J Endod 1982;8:S36-40.

4 Cruse WP, Bellizzi R. A historic review of endodontics, 1689-1963, part 2. J Endod 1980;6:532-5.

5 Bellizzi R, Cruse WP. A historic review of endodontics, 1689-1963, part 3. J Endod 1980;6:576-80.

6 Harrison JW, Baumgartner JC, Svec TA. Incidence of pain associated with clinical factors during and after root canal therapy. Part 2. Postobturation pain. J Endod 1983;9:434-8.

7 Leong DJX, Yap Adrian U-Jin, Yap AU-J. Quality of life of patients with endodontically treated teeth: a systematic review. Aust Endod J 2020;46:130-9

8 Seltzer S, Naidorf IJ. Flare-ups in endodontics: I. etiological factors. $J$ Endod 1985;11:472-8.

9 McKendry DJ. Comparison of balanced forces, endosonic, and stepback filing instrumentation techniques: quantification of extruded apical debris. J Endod 1990;16:24-7.

10 Siqueira JF. Microbial causes of endodontic flare-ups. Int Endod J 2003;36:453-63.

11 Kuștarci A, Akpinar KE, Er K. Apical extrusion of intracanal debris and irrigant following use of various instrumentation techniques. Oral Surg Oral Med Oral Pathol Oral Radiol Endod 2008;105:257-62.

12 Tanalp J, Güngör T. Apical extrusion of debris: a literature review of an inherent occurrence during root canal treatment. Int Endod J 2014;47:211-21.

13 Tanalp J, Kaptan F, Sert S, et al. Quantitative evaluation of the amount of apically extruded debris using 3 different rotary instrumentation systems. Oral Surg Oral Med Oral Pathol Oral Radiol Endod 2006;101:250-7.

14 Moazzami F, Khojastepour L, Nabavizadeh M, et al. Cone-Beam computed tomography assessment of root canal transportation by Neoniti and Reciproc single-file systems. Iran Endod $J$ 2016;11:96-100.

15 Keskin C, Demiral M, Sarıyılmaz E. Comparison of the shaping ability of novel thermally treated reciprocating instruments. Restor Dent Endod 2018;43:e15.

16 Roane JB, Sabala CL, Duncanson MG. The "balanced force" concept for instrumentation of curved canals. J Endod 1985;11:203-11.
17 Siqueira JF, Rôças IN, Favieri A, et al. Incidence of postoperative pain after intracanal procedures based on an antimicrobial strategy. $J$ Endod 2002;28:457-60.

18 Pedrinha VF, Brandão JMdaS, Pessoa OF, et al. Influence of file motion on shaping, apical debris extrusion and Dentinal defects: a critical review. Open Dent J 2018;12:189-201.

19 Gutmann JL, Gao Y. Alteration in the inherent metallic and surface properties of nickel-titanium root canal instruments to enhance performance, durability and safety: a focused review. Int Endod J 2012;45:113-28.

20 Shen Y, Tra C, Hieawy A, et al. Effect of torsional and fatigue Preloading on HyFlex EDM files. J Endod 2018;44:643-7.

21 Azim $\mathrm{AA}$, Wang $\mathrm{HH}$, Tarrosh $\mathrm{M}$, et al. Comparison between single-file rotary systems: part 1-Efficiency, effectiveness, and adverse effects in endodontic retreatment. J Endod 2018;44:1720-4.

22 Shamseer L, Moher D, Clarke M, et al. Preferred reporting items for systematic review and meta-analysis protocols (PRISMA-P) 2015: elaboration and explanation. BMJ 2015;350:g7647.

23 Sollenberger JF, Holloway RG. The evolving role and value of libraries and librarians in health care. JAMA 2013;310:1231-2.

24 Caviedes-Bucheli J, Castellanos F, Vasquez N, et al. The influence of two reciprocating single-file and two rotary-file systems on the apical extrusion of debris and its biological relationship with symptomatic apical periodontitis. A systematic review and meta-analysis. Int Endod J 2016;49:255-70.

25 Faggion CM. Guidelines for reporting pre-clinical in vitro studies on dental materials. J Evid Based Dent Pract 2012;12:182-9.

26 Chapman CE, Collee JG, Beagrie GS. A preliminary report on the correlation between apical infection and instrumentation in endodontics. J Br Endod Soc 1968;2:7-11.

27 Vande Visse JE, Brilliant JD. Effect of irrigation on the production of extruded material at the root apex during instrumentation. J Endod 1975;1:243-6.

28 Borenstein M, Hedges LV, Higgins JPT. Introduction to meta-analysis. John Wiley \& Sons, Ltd, 2009.

29 Faraone SV. Interpreting estimates of treatment effects: implications for managed care. P T 2008;33:700.

30 Cohen J. Statistical power analysis for the behavioral sciences. Routledge, 2013.

31 Cohen J. Statistical power analysis for the behavioural sciences. 2nd edn. Hillsdale, NJ: Lawrence Erlbaum Associates, 1988.

32 Higgins JPT, Thompson SG, Deeks JJ, et al. Measuring inconsistency in meta-analyses. BMJ 2003;327:557-60.

33 Higgins JPT, Thompson SG. Quantifying heterogeneity in a metaanalysis. Stat Med 2002;21:1539-58.

34 Egger M, Davey Smith G, Schneider M, et al. Bias in meta-analysis detected by a simple, graphical test. BMJ 1997;315:629-34. 